\title{
Efectos de dos tipos de Biol Enriquecido, aplicado en diferentes dosis sobre el rendimiento de tallos de Palmito (Bactris Gasipaes H.B.K) Santo Domingo de los Tsáchilas, Ecuador, Diciembre 2008
}

\author{
Autores: \\ Ing. Miriam Recalde Quiroz \\ Ing. Luis Gusqui Vilema \\ Stalin Ramos
}


Res Sun esum sumen

La investigación se desarrolló en la granja experimental de la UTE, Santo Domingo, en una plantación de 12 años de edad, cuyo problema principal es la baja emisión de hijuelos que trae como consecuencia una disminución del rendimiento por hectárea. Para lo cual se planteó como objetivo mejorar la emisión de hijuelos mediante la aplicación de bioles enriquecidos, utilizados en diferentes dosis. Se utilizó un diseño de parcela dos veces divididas, con tres repeticiones, constituidas por parcelas (variedades) y su parcelas (biol y dosis); las variedades correspondían a palmito con espinas y sin espinas, los tipos de bioles empleados fueron de bovino y de porcino, con dosis de 0; 15; 30; y 45 cc $3 /$ l de agua. Las variables evaluadas fueron: Diámetro, altura de hijuelos, número de hijuelos por planta, porcentaje de plantas sin hijuelos y número de hojas por planta, realizando para cada una de ellas un análisis de varianza y los promedios fueron comparados utilizando la prueba de Tukey al 5\% de probabilidad. Antes de la aplicación de bioles, se efectuó un análisis situacional de población de cochinilla a nivel radicular, seguido de aplicaciones de insecticida para el respectivo control. Como resultado se obtuvo que bajo las condiciones de la plantación en estudio, los bioles aplicados en diferentes dosis, influyeron en el diámetro de hijuelos, pero no en la altura de los mismos. El número de hijuelos por planta y el porcentaje de plantas sin hijuelos, durante los cuatro bimestres no se ven influenciados por la aplicación de bioles y solo al final se observa un incremento que no es significativo estadísticamente, pero si muestra mejores resultados que los observados inicialmente. El número de hojas funcionales, no es una variable que se vea afectada por la aplicación de bioles. En conclusión, se requiere mayor tiempo de evaluación para llegar a resultados definitivos que muestren la influencia de los bioles en el mejoramiento de la emisión de hijuelos en palmito, manejando dosis más altas y supliendo requerimientos del cultivo bajo un balance nutricional.

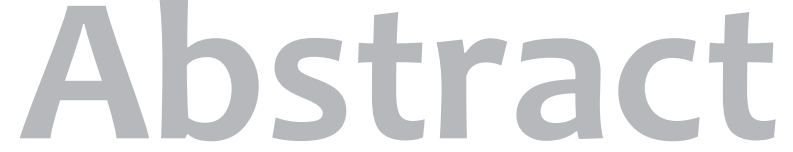

The Palmito research was developed in the Santo Domingo experimental farm of the UTE, in a 12 year old plantation, and its main problem is the low production of offspring resulting in a reduced yield per hectare. For which it was designed to improve the production of offspring through the use of enriched biol used in different doses. The experimental design of two times split pieces of land, which was repeated three times(varieties) and sub pieces of land (biol and dose); the varieties were palmito with prickles and without them, the types of biol used were bovine and pigs, with doses of $0,15,30$, and 45 CC3/ I of water. The variables evaluated were: diameter, height of offspring, offspring number per plant, percentage of plants with no offspring and number of leaves per plant, made for each analysis of variance and the averages were compared using the Tukey test to $5 \%$ probability. Before applying Biol, was a situational analysis of population-level radius cochineal, followed by applications of insecticide to its corresponding control. The result was that under the conditions of the study plantation, applying different doses biol influenced the diameter of offspring, but not in its height. The number of offspring or plant and the percentage of plants without the offspring, during the four quarters are not influenced by the application of biol and only at the end there was an increase which is not statistically significant, but it does show better results than those observed initially. The number of functional leaves is not a variable that is affected by the application of biol. In conclusion, it requires additional time for assessment to reach best results showing the influence of biol to improve the emission of palmito offspring by managing higher doses of the crop requirements and supplying a low nutritional balance. 


\section{Introducción}

Las plantaciones de palmito (Bactris gasipaes H.B.K.) constituyen una forma de uso de la tierra de gran importancia, debido al impacto que tiene en la economía de muchos productores de la zona de Santo Domingo-Ecuador. El éxito del cultivo depende de cuántos hijos pueden llegar a un punto de corte y que estos se distribuyan correctamente en la base del tallo principal, lo ideal sería llegar a obtener 8 a 9 hijos que en la práctica no se observan. Por lo tanto uno de los principales problemas en la producción de palmito dentro de la zona y especialmente en la Universidad Tecnológica Equinoccial es la baja emisión de hijuelos/sitio debido a diferentes factores, entre ellos la baja utilización de sustancias promotoras de crecimiento.

En la Provincia de Santo Domingo hay un promedio de 1500 has de palmito con un rendimiento de 12'000000 tallos/año, y el cultivo sigue en expansión, siendo necesario desarrollar tecnologías que permitan mejorar la producción de tallos/año. Considerando además, que una de las exigencias en el mercado actual es el manejo de una agricultura orgánica, no sólo para garantizar una producción libre de contaminante, sino a la vez para evitar la continua destrucción del medio ambiente; se hace necesario buscar alternativas que permitan mejorar el cultivo utilizando un enfoque orgánico, ya que hasta el momento lo realizado es poco en este área. La utilización de bioles, se muestra como una alternativa viable para el desarrollo del cultivo y que además permite aprovechar la materia orgánica animal disponible en las fincas. Se suma el hecho de que el palmito es un cultivo que tiene varias características que lo hacen atractivo para este tipo de producción, ya que de por si su sistema de manejo es amigable con el ambiente. Y, según Bogantes, 1999, solo un 9\% de la materia seca producida en el campo se extrae con la cosecha o sea que se recicla el 91\% de la biomasa, lo cual contribuye al mejoramiento del suelo. tigación fue:

- Mejorar la emisión de hijuelos por sitio mediante la aplicación de bioles enriquecidos utilizados en diferentes dosis en dos variedades de palmito, para lo cual fue necesario evaluar el efecto de los tipos de biol en la emisión de hijuelos por sitio para cada variedad e identificar la dosis adecuada de aplicación.

\section{Materiales, Métodos y Procedimientos}

La investigación se desarrolló en la finca comercial de palmito de la Universidad Tecnológica Equinoccial, ubicada en Santo Domingo-Ecuador a una altura de $600 \mathrm{msnm}$, con una temperatura media anual de $24,5^{\circ} \mathrm{C}$ y una precipitación de $2800 \mathrm{~mm} /$ año, la zona se clasifica como Bosque Húmedo Tropical (Holdridge, 1978).

El diseño estadístico utilizado fue el de parcela dos veces divididas organizadas en bloques completamente al azar con tres repeticiones; para lo cual, se seleccionaron dos lotes de palmito (variedad 1 sin espinas y 2 con espinas), dentro de los cuales, se aplicaron los dos tipos de bioles en dosis de 0, 15, 30 y 45cc/litro de agua, con una frecuencia mensual, teniendo un total de 16 tratamientos, los cuales se muestran en el cuadro 1; para el efecto, se utilizó una superficie de 9408m2, con un total de 2352 plantas, establecidas a un distanciamiento de 2 × 2m; cada tratamiento estuvo conformado por 49 plantas, dejando una hilera de borde por cada lado, lo que dió una superficie útil que corresponde a 25 plantas/tratamiento; la investigación tuvo una duración de 10 meses, se inició desde la elaboración de los bioles.

Para establecer el efecto de éstos, se registró el incremento en altura y diámetro de los hijuelos; así como el número de hijuelos/ sitio, porcentaje de plantas sin hijuelos, número de hojas/planta con una frecuencia bimestral, utilizando una muestra de cinco plantas/tratamiento. 


\section{Cuadro No 1.}

Tratamientos y nomenclaturas para el ensayo de bioles en palmito (Bactris gasipaes H.B.K.)

\begin{tabular}{|c|c|c|}
\hline Tratamientos & Nomenclatura & Descripción \\
\hline T1 & v1b1do & Variedad 1+biol 1+dosis 0 \\
T2 & v1b1d1 & Variedad 1+biol 1+dosis 1 \\
T3 & v1b1d2 & Variedad 1+biol 1+dosis 2 \\
T4 & v1b1d3 & Variedad 1+biol 1+dosis 3 \\
T5 & v1b2d0 & Variedad 1+biol 2+dosis 0 \\
T6 & v1b2d1 & Variedad 1+biol 2+dosis 1 \\
T7 & v1b2d2 & Variedad 1+biol 2+dosis 2 \\
T8 & v1b2d3 & Variedad 1+biol 2+dosis 3 \\
T9 & v2b1d0 & Variedad 2+biol 1+dosis 0 \\
T10 & v2b1d1 & Variedad 2+biol 1+dosis 1 \\
T11 & v2b1d2 & Variedad 2+biol 1+dosis 2 \\
T12 & v2b1d3 & Variedad 2+biol 1+dosis 3 \\
T13 & Variedad 2+biol 2+dosis 0 \\
\hline T16 & Variedad 2+biol 2+dosis 1 & Vad 2+biol 2+dosis 3 \\
\hline
\end{tabular}

Elaborado por: Recalde Miriam, 2008

\section{- Manejo del experimento}

E Estado de la plantación
El ensayo se realizo en una plantación establecida de palmito de 12 años de edad, con una distancia de siembra de $2 \mathrm{~m}$ entre calle por $1 \mathrm{~m}$ entre plantas dando una densidad de 5000 plantas por hectárea, con un alto porcentaje de plantas faltantes y escaso número de hijuelos por sitio. Además, se debe indicar la alta incidencia de cochinillas (Dysmicoccus brevipes) a nivel radicular y en el tallo.

\section{- Delimitación y etiquetado del área del ensayo}

Inicialmente se delimitó el área total del ensayo y a continuación cada una de las unidades experimentales con la ayuda de estacas marcadas; luego se procedió a la identificación de las parcelas y plantas en estudio con etiquetas, realizando un sorteo al azar de cada uno de los tratamientos.

\section{- Preparación de bioles}

Se realizó con el material orgánico animal (estiércol bovino y porcino) y vegetal (maní forrajero), considerado para la investigación, enriqueciéndolos con elementos minerales en las dosis establecidas para tanque de 200L, como se muestra en el Cuadro No 2. Así mismo, en el Cuadro No 3, se observa la composición de los materiales utilizados en la preparación del biol.

\section{Cuadro No 2.}

Composición del biol enriquecido para el ensayo de palmito (Bactris gasipaes H.B.K.)

\begin{tabular}{|l|l|}
\hline Composición del biol enriquecido & Cantidad \\
\hline Estiércol bovino o porcino & 90 Lbs. bovino o 45 Lbs. porcino \\
Leche & $10 \mathrm{Kg}$ \\
Melaza & $1 \mathrm{~L}$ \\
Roca fosfórica & $1 \mathrm{~L}$ \\
Sulpomag & $2000 \mathrm{~g}$ \\
Boro & $250 \mathrm{~g}$ \\
Manganeso & $200 \mathrm{~g}$ \\
Zinc & $500 \mathrm{~g}$ \\
\hline
\end{tabular}

Fuente: Restrepo, J. 2001

Elaborado por: Recalde Miriam, 2008

Se dejó fermentar por un espacio de 2 meses de acuerdo a lo indicado por Suquilandia, 2002. 


\section{Cuadro No 3.}

Composición química de materiales utilizados para la elaboración de los bioles en la UTE

\begin{tabular}{|l|c|c|c|c|c|c|}
\hline \multicolumn{1}{|c|}{ Fuente } & $\begin{array}{c}\text { Relación } \\
\mathrm{C} / \mathrm{N}\end{array}$ & $\begin{array}{c}\text { Nitrógeno } \\
\%\end{array}$ & $\begin{array}{c}\text { Fosforo } \\
\%\end{array}$ & $\begin{array}{c}\text { Potasio } \\
\%\end{array}$ & Calcio \% & $\begin{array}{c}\text { Magnesio } \\
\%\end{array}$ \\
\hline Estiércol bovino & 4.94 & 1.24 & 0.68 & 0.86 & 2.64 & 1.01 \\
\hline Estiércol porcino & 4.20 & 2.08 & 2.97 & 0.77 & 0.80 & 0.47 \\
\hline Maní forrajero & - & 2.96 & 0.18 & 0.80 & 1.05 & 0.65 \\
\hline${ }^{2}$ Melaza & - & $0.4-1.5$ & $0.6-2.0$ & $2.6-5.0$ & $0.1-1.1$ & - \\
\hline
\end{tabular}

Fuente: Laboratorio de suelos y aguas de la UTE, Santo Domingo

\section{- Mantenimiento de la plantación}

Durante el ensayo las actividades que se realizaron fueron: control manual de malezas con desbrozadora en una frecuencia mensual; y química, utilizando glifosato en dosis de $100 \mathrm{cc} /$ bomba de $20 \mathrm{~L}$, aplicado en las calles; y una continúa limpieza de cepas.

Hay que indicar que se realizaron tres aplicaciones de Diazinon en dosis de $400 \mathrm{cc} / 200 \mathrm{~L}$ de agua para el control de cochinillas presentes en raíces y tallo. $Y$ una fertilización edáfica inicial con formula palmito recomendada por Inaexpo.

Adicionalmente, se realizó un análisis de suelo y foliar tomando como referencia la parcela testigo (do) y la dosis alta (d3) para evaluar el contenido de nutrientes. De igual manera, se realizó el análisis a los estiércoles y bioles como sustento de los resultados obtenidos.

\section{- Aplicación de los bioles}

Para su utilización se preparó en una relación de 1.5, 3 y 4.5 Litros de biol puro por tanque de $100 \mathrm{~L}$ de agua, y posteriormente fue aplicado en drench a todas las plantas en estudio mensualmente, con bomba de mochila de 20 litros de capacidad.

\section{Resultados y Discusión}

\section{Diámetro de hijuelo}

Con resultados obtenidos a nivel de campo, según Tukey 5\%, no se presentan diferencias significativas para variedades en el primer y tercer bimestre evaluado; no así durante la segunda evaluación, donde se observa diferencias significativas, mostrando un mejor incremento en diámetro la variedad dos.

Para la subparcela que corresponde a bioles se encontraron diferencias estadísticas durante el primer y tercer bimestre de evaluación, presentando los mejores promedios el biol 1; durante el segundo bimestre no hubieron diferencias estadísticas.

En cuanto a las dosis no se observó diferencias estadísticas para las evaluaciones realizadas; por lo tanto no influyeron en los promedios obtenidos.

La interacción variedades por biol muestra diferencia estadística durante todo el periodo de la investigación, presentando el mayor incremento en diámetro la variedad dos con el biol 1. Mientras que para la interacción biol-dosis se encontraron diferencias significativas en el primer y tercer bimestre de evaluación, con los mejores promedios para el biol 1 y la dosis tres.

Para la interacción variedades $\mathrm{x}$ biol $\mathrm{x}$ dosis, se presentaron diferentes rangos de significancia en cada una de las evaluaciones realizadas. 
Cuadro No 4.

Incremento bimestral de diámetro de hijuelos en la UTE Santo Domingo

\begin{tabular}{|c|c|c|c|c|}
\hline Tratamientos & Incremento 1 & Incremento 2 & Incremento 3 & Incremento total \\
\hline T1 (v1b1d0) & $0,77 a b c$ & 1,20 cdef & $0,83 \quad$ a & $2,80 \mathrm{abcd}$ \\
\hline T2 (v1b1d1) & $0,73 a b$ & $0,53 a b$ & 0,77 & 2,03 a \\
\hline T3 (v1b1d2) & $0,67 a b$ & 0,73 abcde & 0,62 & 2,02 a \\
\hline T4 (v1b1d3) & $0,80 a b c$ & 0,80 abcde & 0,93 & $2,53 a b$ \\
\hline T5 (v1b2d0) & $0,92 a b c$ & 0,76 abcde & 0,78 & $2,46 a b$ \\
\hline T6 (v1b2d1) & $0,87 a b c$ & 0,73 abcde & 0,93 & $2,53 a b$ \\
\hline $\mathrm{T} 7$ (v1b2d2) & $0,75 a b c$ & 0,47 a & 0,69 & 1,92 a \\
\hline T8 (v1b2d3) & $0,63 a b$ & $0,63 a b c$ & 0,97 & 2,23 a \\
\hline T9 (v2b1d0) & $1,02 \mathrm{bc}$ & $0,67 \mathrm{abcd}$ & 1,63 & 3,32 bcde \\
\hline $\mathrm{T} 10$ (v2b1d1) & $0,84 a b c$ & $1,38 \mathrm{f}$ & 1,62 & 3,84 de \\
\hline $\mathrm{T} 11$ (v2b1d2) & $0,87 a b c$ & 1,31 ef & 1,48 & 3,66 cde \\
\hline $\mathrm{T} 12$ (v2b1d3) & $1,21 \mathrm{c}$ & 1,33 ef & 1,58 & $4,12 \mathrm{e}$ \\
\hline $\mathrm{T} 13$ (v2b2d0) & 0,47 a & 0,97 abcde & 0,60 & 2,06 a \\
\hline $\mathrm{T} 14$ (v2b2d1) & $0,78 a b c$ & 0,72 abcde & 0,87 & $2,37 a b$ \\
\hline $\mathrm{T} 15$ (v2b2d2) & $0,63 a b$ & 1,28 def & 0,84 & $2,76 a b c$ \\
\hline $\mathrm{T} 16(\mathrm{v} 2 \mathrm{~b} 2 \mathrm{~d} 3)$ & $0,60 a b$ & 1,10 bcdef & 0,67 & $2,37 a b$ \\
\hline C.V. $\%$ & 19,24 & 22,07 & 17,03 & 12,54 \\
\hline
\end{tabular}

En el Cuadro No 4, se observa que en el primer bimestre se presentó el mejor incremento en diámetro en el T12 con 1,21cm y el menor corresponde al T13 con 0,47 cm; para el segundo bimestre el mejor fue el T10 $(1,38 \mathrm{~cm})$ seguido por el T12 $(1,33 \mathrm{~cm})$; y para el tercer bimestre de evaluación se presentan diferentes rangos y resultados variables dentro de los tratamientos en estudio.

En el mismo Cuadro, se observa que los promedios de los datos tomados de incremento en diámetro, muestran que el mayor incremento para este variable se presenta para el T12 con $4,12 \mathrm{~cm}$ y el menor en el T13 con 2,06cm.

Los coeficientes de variación, se observan al final de la tabla.
Gráfico No 1.

Representación gráfica de diámetro de hijuelos al inicio y al final en la UTE Santo Domingo

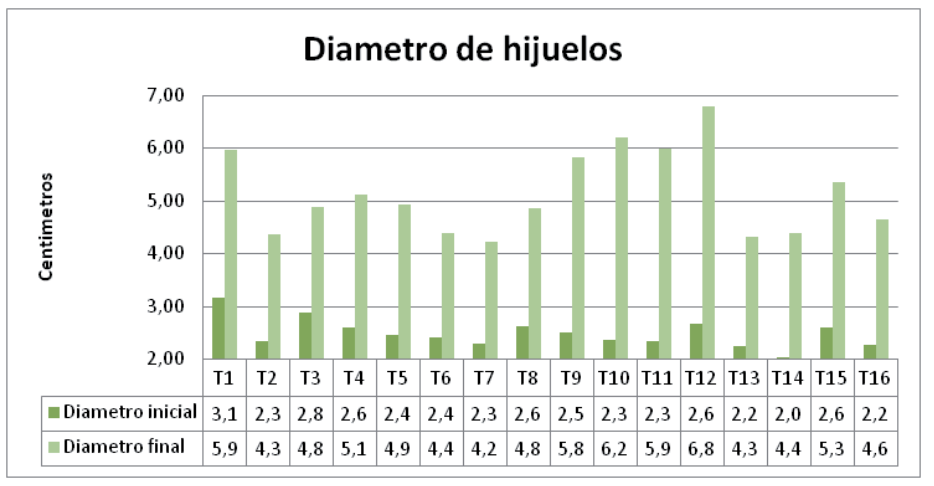

Elaborado por: Recalde, M. 2008

En el Gráfico No 1, se puede observar como el T12 tiene el mayor incremento en diámetro ya que inicio con 2,68cm y alcanzo al final del ensayo $6,80 \mathrm{~cm}(4,2 \mathrm{~cm})$; mientras que el menor incremento fue en el T13 que vario de 2,25 a 4,31cm (2,1cm); analizando estos valores podemos asumir que el biol 1 aplicado en dosis de $4,5 \mathrm{~cm} 3 /$ litro influyo en el diámetro de hijuelos de la variedad de palmito con espinas.

\section{- Altura de hijuelos}

Según resultados obtenidos a nivel de campo para la variable altura de hijuelos, el análisis de varianza muestra diferencias no significativas para la parcela grande que corresponde a variedad con espinas y sin espinas, de igual manera para las subparcelas tipo de biol y dosis; y, para la interacción variedades $\mathrm{x}$ biol $\mathrm{x}$ dosis durante todo el periodo de evaluación. Por lo que se enuncia que los bioles aplicados en diferentes dosis no influyeron en la altura de hijuelos, aunque matemáticamente se puede observar que el menor incremento $(0,30 \mathrm{~cm}) \mathrm{se}$ presentó en el T1 y el mayor en el T11 y T12. (Ver Cuadro 5) 


\section{Cuadro No 5.}

Incremento bimestral de altura de hijuelos (cm) en la UTE Santo Domingo

\begin{tabular}{|c|c|c|c|c|c|c|c|}
\hline \multirow[b]{2}{*}{ Tratamientos } & \multirow{2}{*}{\multicolumn{2}{|c|}{$\begin{array}{c}\text { Incremento } \\
1 \\
\end{array}$}} & \multirow{2}{*}{\multicolumn{2}{|c|}{$\begin{array}{c}\text { Incremento } \\
2 \\
\end{array}$}} & \multirow{2}{*}{\multicolumn{2}{|c|}{ Incremento 3}} & \multirow[b]{2}{*}{ Incremento total } \\
\hline & & & & & & & \\
\hline T1 (v1b1d0) & 0,10 & $a$ & 0,08 & a & 0,12 & a & 0,30 a \\
\hline T2 (v1b1d1) & 0,09 & $a$ & 0,10 & $a$ & 0,12 & a & 0,31 a \\
\hline T3 (v1b1d2) & 0,13 & $a$ & 0,09 & $a$ & 0,09 & a & 0,32 a \\
\hline T4 (v1b1d3) & 0,11 & $\mathrm{a}$ & 0,11 & $a$ & 0,16 & a & 0,37 a \\
\hline T5 (v1b2d0) & 0,11 & $\mathrm{a}$ & 0,14 & $\mathrm{a}$ & 0,10 & a & 0,35 a \\
\hline T6 (v1b2d1) & 0,15 & $\mathrm{a}$ & 0,14 & $\mathrm{a}$ & 0,14 & a & 0,43 a \\
\hline T7 (v1b2d2) & 0,10 & $a$ & 0,13 & $a$ & 0,15 & a & $0,37 a$ \\
\hline T8 (v1b2d3) & 0,15 & a & 0,16 & a & 0,13 & $a$ & 0,44 a \\
\hline T9 (v2b1d0) & 0,11 & a & 0,10 & a & 0,14 & a & $0,34 a$ \\
\hline $\mathrm{T} 10$ (v2b1d1) & 0,13 & $\mathrm{a}$ & 0,15 & $\mathrm{a}$ & 0,13 & $\mathrm{a}$ & 0,41 a \\
\hline $\mathrm{T} 11$ (v2b1d2) & 0,17 & $a$ & 0,12 & $a$ & 0,14 & a & 0,42 a \\
\hline T12 (v2b1d3) & 0,13 & $\mathrm{a}$ & 0,13 & $a$ & 0,15 & $a$ & 0,42 a \\
\hline T13 (v2b2d0) & 0,15 & $a$ & 0,09 & $a$ & 0,10 & a & 0,36 a \\
\hline $\mathrm{T} 14$ (v2b2d1) & 0,09 & $\mathrm{a}$ & 0,11 & $a$ & 0,13 & a & 0,33 a \\
\hline $\mathrm{T} 15$ (v2b2d2) & 0,13 & $a$ & 0,11 & $a$ & 0,09 & a & 0,32 a \\
\hline $\mathrm{T} 16$ (v2b2d3) & 0,09 & $a$ & 0,11 & $a$ & 0,14 & $a$ & 0,36 a \\
\hline C.V. \% & 23,56 & & 25,01 & & 20,96 & & 14,89 \\
\hline
\end{tabular}

Elaborado por: Recalde, M. 2008

\section{Gráfico No 2.}

Representación grafica de altura de hijuelos al inicio y al final en la UTE Santo Domingo

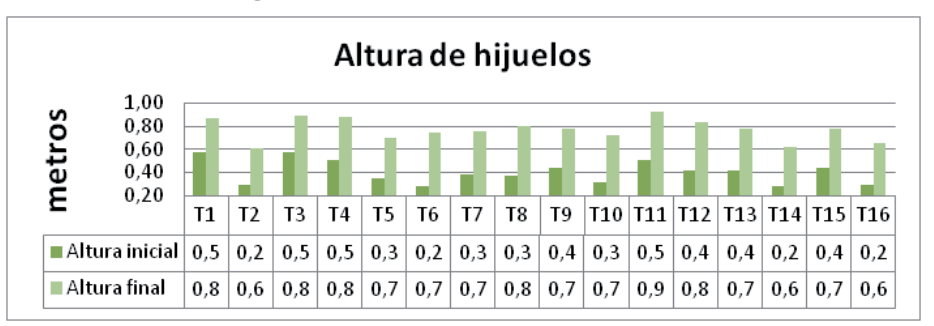

Elaborado por: Recalde, Miriam. 2008

En el Gráfico No 2, se observa la altura inicial y final presentada en cada uno de los tratamientos, donde se muestra que la altura inicial vario entre $0,28 \mathrm{~cm}$ - 0,57cm; y la final de 0,62 en el T14 a 0,92 en el T11; sin embargo el mayor incremento se mostró en los tratamientos T11 y T12 con $0,42 \mathrm{~cm}$.

Pero, estas diferencias estadísticamente no han sido significa- tivas. Por lo tanto, los Bioles aplicados en diferentes dosis no influyeron significativamente en esta variable.

\section{- Número de hijuelos por planta}

Con los resultados obtenidos a nivel de campo y por medio del análisis de varianza, se presentaron diferencias significativas durante el primer bimestre de evaluación para la parcela correspondiente a variedades, mostrando un mejor promedio la variedad sin espinas.

Para las demás evaluaciones no se observaron diferencias estadísticas. En cuanto a la subparcela tipos de biol, se observaron diferencias entre los tratamientos en el segundo y cuarto bimestre; para las otras dos evaluaciones no hubo diferencias notables. En la subparcela dosis, no se registraron diferencias significativas en las evaluaciones realizadas. Se presentaron diferencias en la interacción variedades $x$ biol durante el segundo bimestre, mas no para las otras evaluaciones. En la interacción variedades $\mathrm{x}$ biol $\mathrm{x}$ dosis los promedios obtenidos se presentaron con diferentes rangos durante los tres primeros bimestres; y al final solo mostraron un rango de significancia (Cuadro No 6)

\section{Cuadro No 6.}

Número de hijuelos en la UTE. Santo Domingo

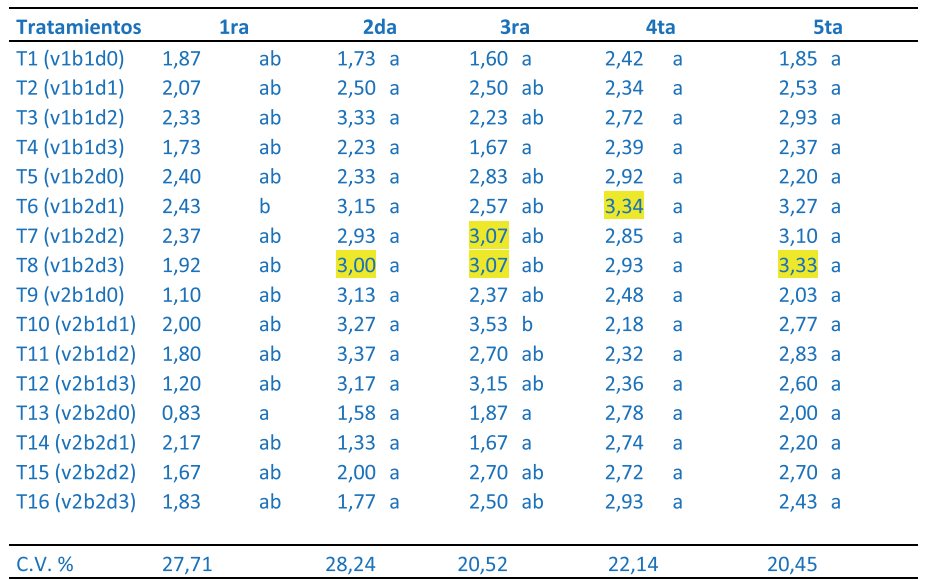

Elaborado por: Recalde, M. 2008 
En el Cuadro No 6, se observa que para el primer bimestre de evaluación, el T13 y el T6 muestran el menor y mayor número de hijuelos respectivamente ( 0,83 y 2,43); para el segundo bimestre no existe diferencias estadísticas entre los tratamientos, sin embargo el menor promedio se presenta en el T14 con 1,33 y el mayor en el T11 con 3,37.

Durante el tercer bimestre evaluado, los rangos son diferentes y el menor número de hijuelos se presentó en el T1 con 1,50; mientras que el mejor es el T10 seguido por el T12, con 3,53 y 3,13.

No se observaron diferencias estadísticas durante la cuarta y quinta evaluación, pero el mejor promedio para esta variable se observo en el T6 con un promedio de 3.34 y el T8 con 3.33 respectivamente. En base a estos resultados, se considera que los bioles aplicados en diferentes dosis en las dos variedades de palmito, no han mostrado una tendencia continua que indique cual es el mejor y en que dosis para esta variable durante la investigación realizada. Pero así mismo, se debe enunciar que los promedios observados en las ultimas evaluaciones son mejores y con mayor homogeneidad que los presentados en la primera evaluación.

\section{- Porcentaje de plantas sin hijuelos}

El análisis de varianza no muestra diferencias estadísticas para la parcela correspondiente a variedades durante el periodo de la investigación; en lo que se refiere a la subparcela tipos de biol se presentan diferencias estadísticas en el segundo, tercero y cuarto bimestre de evaluación. Al evaluar la subparcela dosis, se observa diferencia únicamente en el segundo bimestre, los demás muestran un rango de significancia.

Para la interacción variedades $\mathrm{x}$ dosis, no existe diferencias estadísticas, pero si en la interacción variedades x biol, donde se presenta menor porcentaje de plantas sin hijuelos en la variedad sin espinas con el biol bovino, durante los tres bimestres últimos. tran diferentes rangos de significancia en cada una de las evaluaciones realizadas durante el periodo de investigación.

En el Cuadro No 7, se observa que durante el primer y segundo bimestre los resultados varían para cada uno de los tratamientos en estudio y algo similar se presenta durante la tercera evaluación. Pero durante el cuarto bimestre el T3, T4 y T8 presentan los porcentajes más bajos de plantas sin hijuelos (20\%) e igualmente el mayor porcentaje se ubica en el T9 (66,6\%). De igual manera es notable que al comparar los resultados de la primera evaluación con la última, en forma general existe una disminución del porcentaje plantas sin hijuelos.

Si bien es cierto, estos promedios no muestran específicamente un tratamiento como el mejor, si se debe indicar que la aplicación de bioles en diferentes dosis para las dos variedades influye en la disminución del porcentaje de plantas sin hijuelos, pero se necesita mayor espacio de tiempo para corroborar los resultados en mención.

\section{Cuadro No 7.}

Promedio bimestral del Porcentaje de plantas sin hijuelos (\%) en la UTE Santo Domingo

\begin{tabular}{|c|c|c|c|c|c|c|c|}
\hline \multirow{2}{*}{$\begin{array}{l}\text { Tratamientos } \\
\mathrm{T} 1 \text { (v1b1d0) }\end{array}$} & \multicolumn{2}{|c|}{$1 \mathrm{ra}$} & $2 \mathrm{da}$ & \multicolumn{2}{|c|}{$3 r a$} & \multicolumn{2}{|c|}{4 ta } \\
\hline & 40,00 & $a b$ & $33,30 a b$ & 20,00 & $a b c$ & 26,67 & $a b$ \\
\hline T2 (v1b1d1) & 33,30 & $a b$ & $33,30 a b$ & 6,67 & $\mathrm{a}$ & 26,67 & $a b$ \\
\hline T3 (v1b1d2) & 40,00 & $a b$ & 13,30 a & 13,33 & $a b$ & 20,00 & a \\
\hline T4 (v1b1d3) & 20,00 & $a b$ & 6,67 a & 20,00 & $a b c$ & 20,00 & a \\
\hline T5 (v1b2d0) & 40,00 & $a b$ & $26,67 a b$ & 53,33 & abcd & 40,00 & $a b$ \\
\hline T6 (v1b2d1) & 13,33 & a & $20,00 a b$ & 26,67 & abcd & 40,00 & $a b$ \\
\hline T7 (v1b2d2) & 40,00 & $a b$ & $46,67 a b$ & 20,00 & $a b c$ & 33,30 & $a b$ \\
\hline T8 (v1b2d3) & 20,00 & $a b$ & $33,30 a b$ & 20,00 & $a b c$ & 20,00 & $\mathrm{a}$ \\
\hline T9 (v2b1d0) & 60,00 & $a b$ & $46,67 a b$ & 26,67 & abcd & 66,67 & $b$ \\
\hline $\mathrm{T} 10$ (v2b1d1) & 26,67 & $a b$ & $26,67 a b$ & 20,00 & $a b c$ & 46,67 & $a b$ \\
\hline T11 (v2b1d2) & 40,00 & $a b$ & $60,00 a b$ & 33,30 & abcd & 40,00 & $a b$ \\
\hline T12 (v2b1d3) & 26,67 & $a b$ & $33,30 a b$ & 40,00 & abcd & 33,30 & $a b$ \\
\hline T13 (v2b2d0) & 73,33 & $b$ & $46,67 a b$ & 60,00 & $\mathrm{~cd}$ & 53,30 & $a b$ \\
\hline T14 (v2b2d1) & 46,67 & $a b$ & $80,00 \quad b$ & 66,67 & d & 46,60 & $a b$ \\
\hline $\mathrm{T} 15$ (v2b2d2) & 46,67 & $a b$ & $46,67 a b$ & 33,30 & abcd & 20,00 & a \\
\hline T16 (v2b2d3) & 73,33 & $b$ & $60,00 a b$ & 46,60 & abcd & 26,67 & $a b$ \\
\hline C.V. \% & 21,61 & & 26,15 & 29,93 & & 20,29 & \\
\hline
\end{tabular}

Elaborado por: Recalde, M. 2008

En la interacción variedades $\mathrm{x}$ biol x dosis, los resultados mues- 


\section{- Número de hojas por planta}

Adicionalmente, se evaluó el número de hojas funcionales por planta, mostrando en el segundo bimestre diferencias significativas para las variedades y para tipos de biol, en las otras evaluaciones no hubo diferencias; igualmente no se presentó diferencias para la parcela correspondiente a dosis.

En la interacción variedades $x$ biol se presentan diferencias en el segundo y tercer bimestre de evaluación; mientras que en la interacción variedades $\mathrm{x}$ dosis no existe diferencia estadística en las evaluaciones realizadas.

En el Cuadro No 8, se observa los promedios de la interacción variedades $\mathrm{x}$ biol $\mathrm{x}$ dosis, pudiendo notarse que durante el primero $\mathrm{y}$ cuarto bimestre no hay diferencias entre los tratamientos en estudio, que presentaron entre 3,20 y 4,60 (T14 y T6); 3,14 y 4,58 (T14 y T9) hojas/planta, respectivamente; mientras que en el segundo y tercer bimestre de evaluación se presentaron dos rangos de significancia. Estos resultados demuestran que la aplicación de bioles en diferentes dosis no influye en el número de hojas por planta.

\section{Cuadro No 8.}

Promedio bimestral del Número de hojas por planta en la UTE Santo

\section{Domingo}

\begin{tabular}{|c|c|c|c|c|c|c|}
\hline \multirow{2}{*}{$\begin{array}{l}\text { Tratamientos } \\
\mathrm{T} 1 \text { (v1b1d0) }\end{array}$} & \multicolumn{2}{|c|}{$1 \mathrm{ra}$} & \multirow{2}{*}{$\frac{2 \mathrm{da}}{4,17 \mathrm{ab}}$} & \multirow{2}{*}{$\frac{3 \mathrm{ra}}{3,93 \mathrm{ab}}$} & \multicolumn{2}{|c|}{4 ta } \\
\hline & 4,07 & $\mathrm{a}$ & & & 3,28 & a \\
\hline T2 (v1b1d1) & 4,33 & a & $3,93 a b$ & $3,87 a b$ & 4,18 & a \\
\hline T3 (v1b1d2) & 4,27 & a & $4,67 a b$ & $4,20 a b$ & 3,15 & a \\
\hline T4 (v1b1d3) & 3,93 & a & $4,37 a b$ & $3,83 a b$ & 4,13 & a \\
\hline T5 (v1b2d0) & 4,50 & a & $4,50 a b$ & $4,50 a b$ & 4,31 & a \\
\hline T6 (v1b2d1) & 4,60 & a & $3,73 a b$ & $4,03 a b$ & 4,14 & a \\
\hline $\mathrm{T} 7$ (v1b2d2) & 4,63 & a & $4,00 a b$ & $5,17 \mathrm{~b}$ & 4,18 & a \\
\hline T8 (v1b2d3) & 4,27 & a & $4,40 a b$ & $3,83 a b$ & 4,26 & a \\
\hline T9 (v2b1d0) & 3,27 & a & $4,40 a b$ & $4,43 a b$ & 4,58 & a \\
\hline T10 (v2b1d1) & 3,67 & a & 5,27 b & $4,33 a b$ & 4,14 & a \\
\hline T11 (v2b1d2) & 3,60 & a & $4,27 a b$ & $4,33 a b$ & 4,42 & a \\
\hline T12 (v2b1d3) & 3,53 & a & $4,43 a b$ & $4,03 a b$ & 3,80 & a \\
\hline $\mathrm{T} 13$ (v2b2d0) & 3,70 & a & $3,80 a b$ & 3,20 a & 3,44 & a \\
\hline T14 (v2b2d1) & 3,67 & $a$ & 3,20 a & 3,13 a & 3,14 & a \\
\hline T15 (v2b2d2) & 4,20 & a & $3,80 a b$ & $3,90 \mathrm{ab}$ & 3,85 & a \\
\hline T16 (v2b2d3) & 3,80 & a & 3,60 a & $3,47 a b$ & 3,41 & a \\
\hline CV\% & 13,09 & & 12,31 & 14,6 & 18,8 & \\
\hline
\end{tabular}

\section{- Número de tallos cosechados}

El análisis de varianza presenta diferencias significativas para la parcela grande que corresponde a variedades, siendo la variedad sin espinas la que muestra un mayor número de tallos. Igualmente, se observó diferencias estadísticas para la subparcela correspondiente a bioles. En cuanto a la interacción variedades $x$ biol, biol $x$ dosis y variedad $\mathrm{x}$ dosis, no se presentaron diferencias significativas.

En el Cuadro No 9, se observa los promedios por tratamiento del número de tallos cosechados, pudiendo notar que el menor promedio se encuentra en el T10 (1600 tallos) y el mayor en el T3 (4800 tallos). A pesar de que existen diferentes rangos de significancias, no se podría asumir que se debe al efecto de los bioles. Ya que se hizo una proyección del rendimiento en base a dos meses de cosecha.

\section{Cuadro No 9.}

Número de tallos cosechados por tratamiento en la UTE, Santo Domingo

\begin{tabular}{lll} 
Tratamientos & \multicolumn{2}{l}{ N $^{\circ}$ Tallos/Ha/año } \\
\hline T1 (v1b1d0) & 4000,00 & $a b$ \\
T2 (v1b1d1) & 4400,00 & $b$ \\
T3 (v1b1d2) & 4800,00 & $b$ \\
T4 (v1b1d3) & 4400,00 & $b$ \\
T5 (v1b2d0) & 3200,00 & $a b$ \\
T6 (v1b2d1) & 4400,00 & $b$ \\
T7 (v1b2d2) & 2800,00 & $a b$ \\
T8 (v1b2d3) & 3600,00 & $a b$ \\
T9 (v2b1d0) & 4000,00 & $a b$ \\
T10 (v2b1d1) & 1600,00 & $a$ \\
T11 (v2b1d2) & 3200,00 & $a b$ \\
T12 (v2b1d3) & 3200,00 & $a b$ \\
T13 (v2b2d0) & 2800,00 & $a b$ \\
T14 (v2b2d1) & 1600,00 & $a$ \\
T15 (v2b2d2) & 3600,00 & $a b$ \\
T16 (v2b2d3) & 3200,00 & $a$ \\
\hline Cv\% & & \\
\hline
\end{tabular}


Los resultados se presentan en el Cuadro No 10, donde se ob-

En el Cuadro No11, se presentan los resultados de los análisis serva que el análisis de suelo realizado en el cultivo de palmito don-químicos realizados a los bioles utilizados en el ensayo, pudiendo obde se aplicó el B1 y el B2, en diferentes dosis presenta un porcentajeservar que la relación carbono nitrógeno es mayor para el biol bovino. de Materia orgánica entre 4.60 y 4.73 considerado como nivel medio,Así mismo, se muestra que el contenido de nitrógeno es bajo, en relacercano al óptimo para el cultivo. El pH observado está entre 5.51 yción a lo indicado por Suquilandia, 2003, quien presenta datos de 1,6\%, 5.63, aceptado dentro de los requerimientos del cultivo. Por lo tantoen biol bovino, influenciado posiblemente por el tipo de alimentación, no ha sido influenciado por las aplicaciones de biol. Y es mayor que elbasado en leguminosas. De igual forma, se observa que los demás elereportado por TUTILLO, M. en un análisis de suelo de una plantaciónmentos presentan un contenido bajo, lo cual es aceptable, debido a de palmito ubicada en el Cortijo II, Santo Domingo. que el biol no es una fuente de elementos minerales, sino de fitore-

En cuanto a macro elementos, se observó un nivel bajo de niguladores. trógeno (24.18 y 27.26) e igualmente para Potasio con 0.28 y 0.17. LosCuadro No 11. demás elementos se encuentran dentro de los parámetros óptimos.

\section{Composición química de bioles}

\section{Cuadro No 10.}

Comparación de las características optimas de suelo para e cultivo de palmito con el resultado de análisis de suelo de los dos bioles.

\begin{tabular}{|c|c|c|c|c|c|c|}
\hline Fuente & $\begin{array}{c}\text { Relación } \\
\mathrm{C} / \mathrm{N}\end{array}$ & $\begin{array}{c}\text { Nitrógeno } \\
\%\end{array}$ & Fosforo \% & $\begin{array}{c}\text { Potasio } \\
\%\end{array}$ & Calcio \% & $\begin{array}{c}\text { Magnesio } \\
\%\end{array}$ \\
\hline Biol bovino & 5.88 & 0.01 & 0.01 & 0.09 & 0.23 & 0.04 \\
\hline Biol porcino & 2.0 & 0.05 & 0.02 & 0.11 & 0.23 & 0.03 \\
\hline
\end{tabular}

Elaborado por: Recalde Miriam, 2008

\begin{tabular}{|c|c|c|c|}
\hline \multirow[b]{2}{*}{ Características } & \multirow[b]{2}{*}{$\begin{array}{l}\text { Valor } \\
\text { optimo }^{1}\end{array}$} & \multicolumn{2}{|c|}{$\begin{array}{l}\text { Resultado de análisis }{ }^{2} \\
\text { Cultivo de palmito } \\
\text { UTE }\end{array}$} \\
\hline & & $\begin{array}{c}\text { Trat. Biol } \\
1\end{array}$ & $\begin{array}{c}\text { Trat. Biol } \\
2\end{array}$ \\
\hline $\mathrm{pH}(\mathrm{H} 2 \mathrm{O})$ & $5.5-6.0$ & 5.63 & 5.51 \\
\hline Materia orgánica \% & $>5$ & 4.60 & 4.73 \\
\hline Fosforo ppm & $>10$ & 26.94 & 41.76 \\
\hline $\begin{array}{l}\text { Calcio meq/100g } \\
\text { Magnesio }\end{array}$ & $>4$ & 8.47 & 8.30 \\
\hline $\mathrm{meq} / 100 \mathrm{~g}$ & $>1.0$ & 1.37 & 1.04 \\
\hline Potasio meq/100g & $>0.3$ & 0.28 & 0.17 \\
\hline Aluminio & & & \\
\hline meq/100g & $<1.0$ & - & - \\
\hline Azufre ppm & $>10$ & - & - \\
\hline Hierro ppm & 10 a 50 & 230.0 & 155.2 \\
\hline Manganeso & 5 a 50 & 6.30 & 6.00 \\
\hline Cobre ppm & 1 a 10 & 7.50 & 8.10 \\
\hline Zinc ppm & 3 a 15 & 8.90 & 21.5 \\
\hline Boro ppm & 0.5 a 2 & 0.99 & 1.09 \\
\hline
\end{tabular}

\section{- Costos del experimento}

Los costos del experimento, se detallan en el Cuadro No 12, donde se ha separado costos de infraestructura y de preparación de bioles, durante los diez meses que duro la evaluación. En este cuadro, se observa que en materiales utilizados para los bioles hay un costo de $\$ 85,11$ y en materia prima necesaria para la preparación $\$ 110,89$, lo cual $\frac{m}{\sigma}$ da un total de $\$ 196$ que corresponden a 10 tanques de biol; por lo tanto cada uno tendría un costo de $\$ 19,6 /$ tanque de $100 \mathrm{~L}$ de biol puro. 


\section{Cuadro No 12.}

\section{Costos relacionados con infraestructura y preparación de bioles}

\begin{tabular}{|c|c|c|c|c|c|c|}
\hline & Cantidad & Unidad & $\begin{array}{c}\text { Precio US } \\
\$\end{array}$ & $\begin{array}{l}\text { Precio } \\
\text { Total } \$\end{array}$ & $\begin{array}{l}\text { Deprec. } \\
\text { Años }\end{array}$ & Total \\
\hline \multicolumn{7}{|l|}{ Recursos Humanos } \\
\hline Jornales & 20 & & 10 & 200 & & 200 \\
\hline \multicolumn{7}{|l|}{ Materiales } \\
\hline Tubos de metal & 10 & & 44.8 & 448 & 10 & 37,33 \\
\hline Hojas de zinc & 47 & & 8.99 & 422,86 & 5 & 70,47 \\
\hline Cañas & 35 & & 1.61 & 56,56 & 2 & 23,6 \\
\hline Clavos de zinc & 5 & Lbs. & 0.998 & 4,99 & 2 & 2,08 \\
\hline Sub total & \multicolumn{5}{|c|}{932,41} & 333,48 \\
\hline \multicolumn{7}{|l|}{ Ostos materiales para biol } \\
\hline Tanques de $200 \mathrm{~L}$ & 10 & & 22.08 & 220,09 & 5 & 36,68 \\
\hline Conector para tanque $1 / 2$ & 10 & & 1.8 & 18 & 1 & 18 \\
\hline Adaptador flex $1 / 2$ & 10 & & 0.24 & 2,4 & 1 & 2,4 \\
\hline $\begin{array}{l}\text { Manguera lisa transparente } \\
1 / 2\end{array}$ & 13 & $\mathrm{~m}$ & 0.25 & 3,25 & 1 & 3,25 \\
\hline Guantes ginecológicos & 25 & & 0.18 & 4,5 & 1 & 4,5 \\
\hline Guantes de caucho & 2 & pares & 1.25 & 2,1 & 1 & 2,1 \\
\hline plástico negro & 10 & $m$ & 1.0 & 10 & 1 & 10 \\
\hline Teflón & 3 & & & 0,9 & 1 & 0,9 \\
\hline Abrazaderas & 10 & & 0.268 & 2,68 & 1 & 2,68 \\
\hline Cinta de embalaje & 3 & & 1.0 & 3 & 1 & 3 \\
\hline Mascarillas & 4 & & 0.40 & 1,6 & 1 & 1,6 \\
\hline \multicolumn{4}{|l|}{ SUB TOTAL } & 268,52 & 1 & 85,11 \\
\hline \multicolumn{5}{|c|}{ Costos de reparaciones de bioles } & $\begin{array}{l}\text { Uso en } \\
\text { meses }\end{array}$ & \\
\hline Recursos humanos & 2 & jornales & 7 & 14 & & 14 \\
\hline Porcino & 225 & Lbs. & & 5 & 2 & 5 \\
\hline Bovino & 450 & Lbs. & & 10 & 2 & 10 \\
\hline Trazex zinc & 2.5 & $\mathrm{Kg}$ & 16,32 & 40,8 & 2 & 40,8 \\
\hline Roca fosfórica & 20 & $\mathrm{Kg}$ & 0,36 & 7,2 & 2 & 7,2 \\
\hline Bórax & 2 & $\mathrm{Kg}$ & 0,91 & 1,82 & 2 & 1,82 \\
\hline Sulpomag & 2,5 & $\mathrm{Kg}$ & 0,53 & 1,32 & 2 & 1,32 \\
\hline Manganeso & 5 & $\mathrm{~L}$ & 7,25 & 36,25 & 2 & 36,25 \\
\hline Melaza & 10 & $\mathrm{~L}$ & 0.35 & 3,5 & 2 & 3,5 \\
\hline Leche & 10 & $\mathrm{~L}$ & 0.50 & 5,0 & 2 & 5,0 \\
\hline \multicolumn{4}{|l|}{ Sub total } & & & 110,89 \\
\hline
\end{tabular}

\section{Conclusiones y Recomendaciones}

\section{Conclusiones}

1. La aplicación de bioles en diferentes dosis influyo en el diámetro de hijuelos, con un incremento de $4,12 \mathrm{~cm}$ obtenido con dosis de $4,5 \mathrm{~cm} 3 / \mathrm{L}$; mientras que no ejerce influencia alguna en la altura de hijuelos durante el periodo de evaluación de la plantación en estudio.

2. Las dosis aplicadas de los dos bioles no presentaron diferencias en el número de hijuelos de las dos variedades de palmito, y solo al final se observa una homogeneidad de los promedios, con respecto a las primeras evaluaciones; por lo tanto se requiere mayor tiempo de evaluación para llegar a resultados definitivos.

3. El porcentaje de plantas sin hijuelos disminuyo al final de la evaluación con la aplicación de bioles, sin embargo no se determino el mejor tratamiento para esta plantación.

4. El número de hojas por planta, no es una variable que se vea afectada por la aplicación de bioles.

5. Los resultados de los análisis de suelo realizados en las parcelas donde se aplicaron los bioles, mostraron valores bajos de Nitrógeno y Potasio, los demás elementos se encuentran en cantidades óptimas de acuerdo a los requerimientos del cultivo.

6. Considerando que es una plantación con problemas marcados de producción, la aplicación de bioles es insuficiente para ver resultados satisfactorios en el periodo establecido de evaluación.

7. El costo de un tanque de biol de $100 \mathrm{~L}$ es de $\$ 19,6$; por lo tanto resulta barato producir este preparado. Tomando en cuenta que los tanques plásticos utilizados, se deprecian en 5 años de uso.

\section{Recomendaciones}

1. Para lograr mejores resultados en la aplicación de los dos bioles se requiere mayor tiempo de evaluación y así lograr llegar a resultados definitivos.

2. Se recomienda además de la aplicación de bioles en plantaciones con problemas marcados de producción, considerar otros tratamientos que permitieran lograr resultados satisfactorios.

3. Se recomienda una fertilización completa balanceada en base a los requerimientos del cultivo, donde el biol sea un complemento.

4. Por el bajo costo del tanque de biol y su duración, se sugiere sean utilizados en la región.

5. Realizar investigación manejando dosis más altas de biol considerando en base a porcentajes de la siguiente forma: 25,50 , 75 y $100 \%$ de biol puro. 


\section{Bibliografía consultada}

1. Burneo, J. Bioway, 1997. Información técnica, su utilización en la Agricultura, Acuacultura y Ganadería, INAEXPO, Quito Ecuador Pp. 35-38.

2. Bogantes, A. 1997. Evaluación de dos sustratos en plantas de Pejibaye (Bactris gasipaes) en vivero. Informe final. Estación Experimental Los Diamantes.

3. Chala, V. 1996. Manejo del cultivo de palmito, Proyecto MAGFAD, Santo Domingo- Ecuador Pp. \&,7.

4. Molina, E. 2000. Manual de suelos y nutrición de pejibaye para palmito. Centro de Investigaciones agronómicas. Universidad de Costa Rica.

5. ------ PROEXANT, 1992. Manual del cultivo de Palmito, QuitoEcuador Pp. 1-11.

6. Restrepo, J. 2001. Elaboración de abonos orgánicos fermentados y biofertilizantes foliares. 155p.

7. Rojas, E. 1999. Suelos, Nutrición Mineral y Fertilización. Palmito de Pejibaye, San José-Costa Rica.

8. Suquilanda, M. 2003. Agricultura orgánica. Alternativa Tecnológica del futuro. Pp 219-240.

\section{La Investigadora}

Miriam Recalde Quiroz

mrecalde@ute.edu.ec

Ingeniera Forestal; Universidad

Técnica de Quevedo, 2000

Maestría en Nutrición Vegetal, Universidad Tecnológica Equinoccial 


\section{Anexos}

\section{ANEXO 1. Medición de diámetro del tallo}

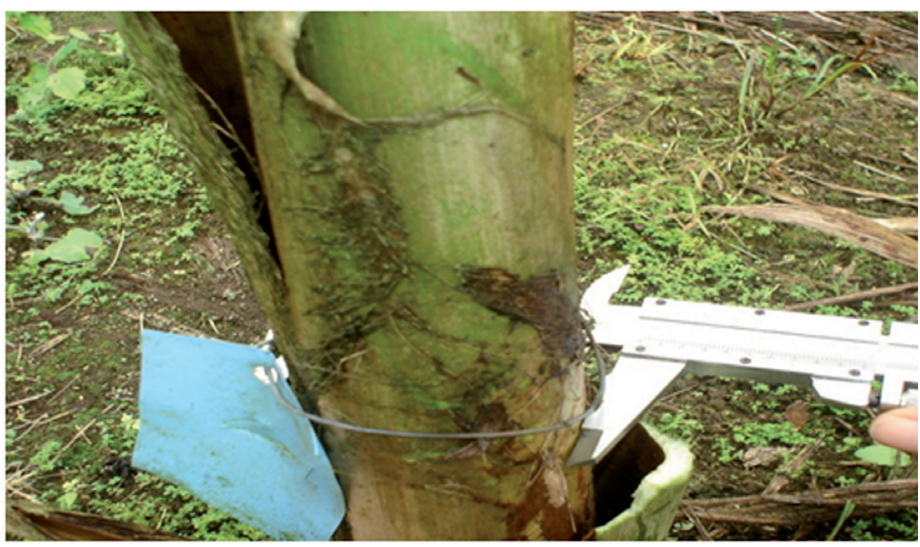

\section{ANEXO 2. Medición de altura de hijuelos}

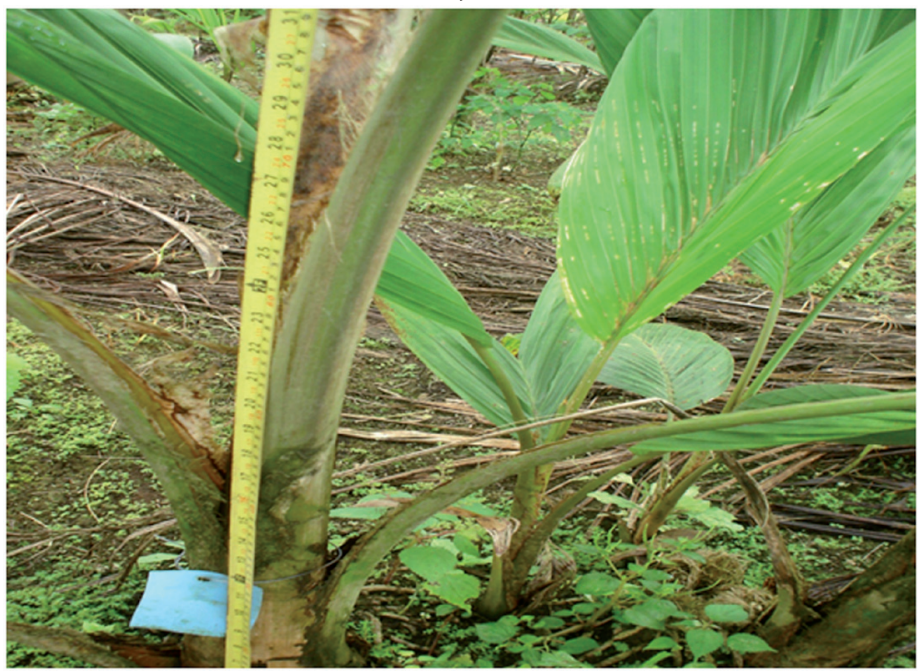

\section{ANEXO 3: Conteo de número de hijuelos}

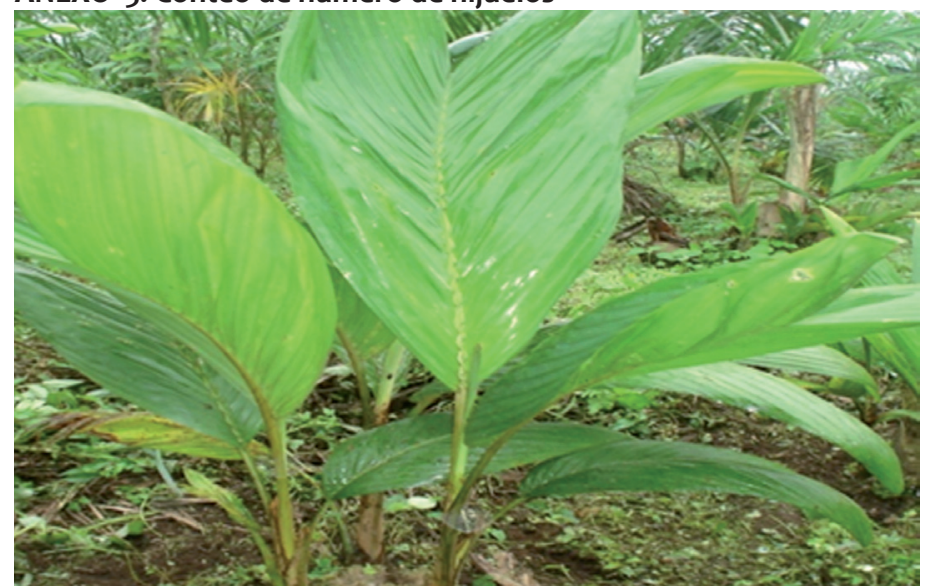

\section{ANEXO 4: Etiquetado de plantas}

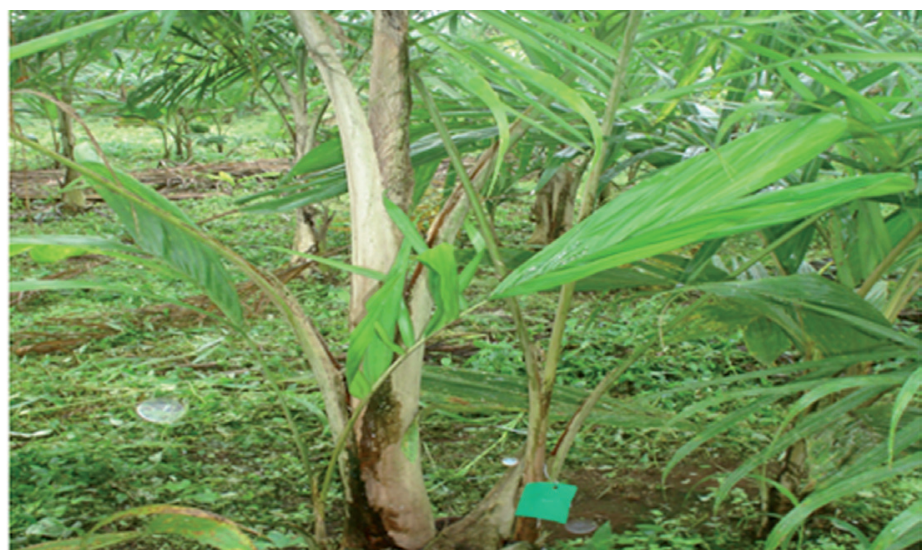

\section{ANEXO 5. Manejo de la plantación}

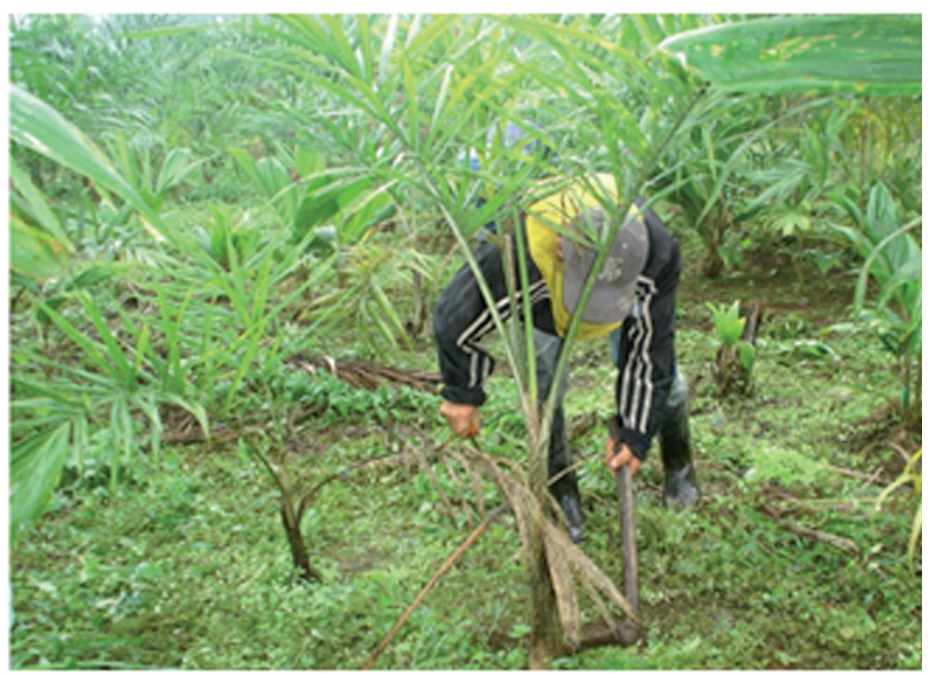

